\title{
Excited State Dynamics with Quantum Trajectories
}

\author{
Basile F. E. Curchod§, Ursula Rothlisberger, and Ivano Tavernelli* \\ §SCS-Metrohm Foundation Award for best oral presentation
}

\begin{abstract}
Nuclear quantum dynamics beyond the Born-Oppenheimer approximation is performed using quantum trajectories. Within the adiabatic representation of the electronic states, NABDY (NonAdiabatic Bohmian DYnamics) is used in combination with DFT and LR-TDDFT to perform on-the-fly nonadiabatic quantum dynamics. Simple numerical test systems and current limitations of the method are discussed.
\end{abstract}

Keywords: Born-Oppenheimer approximation - Linear-response time-dependent density functional theory . Nonadiabatic dynamics · Quantum trajectories

\section{Introduction}

The Born-Oppenheimer approximation (BOA) constitutes a cornerstone in the implementation of quantum mechanics for chemical systems. By neglecting the couplings between electronic states due to the motion of the nuclei, this method has paved the way to development of ground state electronic structure methods for molecules. In addition, the BOA allows for efficient mixed quantum/classical schemes to perform ab initio molecular dynamics ${ }^{[1]}$ (AIMD), where electrons are treated quantum mechanically and provide the potential for the classical dynamics of the nuclei. Considerable computational savings are indeed obtained when only one electronic state is considered and the BOA has therefore made possible the simulation of large molecules for several picoseconds.

However, as soon as more than one electronic state becomes important for a particular chemical process (e.g. for a photochemical reaction), the BOA is likely to fail due to the coupling between different electronic states. [2] In fact, when a nuclear wavepacket representing the molecular system of interest is evolving on an electronic state and approaching a conical intersection or an avoided crossing, there is a growing probability that part of its amplitude is transferred to a second state (see Fig. 1a,b for a pictorial representation of such an event). This event is the result of the coupling between electronic states, induced by the nuclear motions and described by the nonadiabatic couplings of first and second order ${ }^{22}$ (see Section 2 below for mathematical expressions).

Nuclear dynamics on excited states can be accurately described by using nuclear wavepacket propagation methodologies, like for example multiconfiguration timedependent Hartree (MCTDH). ${ }^{[3]}$ Even though this method offers a very accurate description of the nuclear propagation, it is hampered by the limited number of degrees of freedom that it can handle and by the need of precomputed potential energy surfaces (PESs). On the other hand, nonadiabatic events are usually difficult to describe by means of standard trajectory-based methods and therefore only approximate solutions of this dynamics are currently used. Among them, in Ehrenfest dynamics the total electronic wavefunction of the system is propagated in time, producing a mean-field potential for the motion of the classical nuclei. Even though the resulting nuclear trajectory has no clear physical meaning, it can be associated to the center of mass motion of the moving nuclear wavepacket.

Another trajectory-based solution of the excited state dynamics is the so-called 'trajectory surface hopping' (TSH) approach. In its most successful implementation, ${ }^{[4]}$ TSH portrays the nuclear wavepacket as a swarm of independent classical trajectories, which can hop from one PES

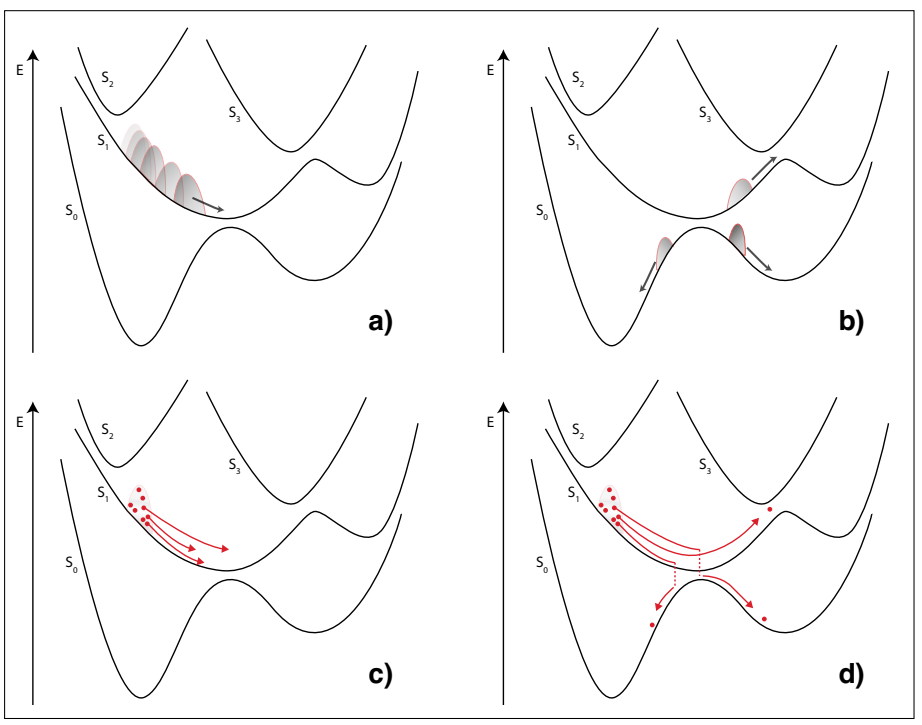

Fig. 1. Schematic representation of an exact nuclear wavepacket propagation (upper part) and TSH (lower part) before (a and $\mathbf{c}$ ) and after (b and d) a nonadiabatic event. Red dots represent different initial conditions and red arrows the corresponding different trajectories. 
to another whenever strong nonadiabaticity is experienced by the molecular system. The occurrence of a trajectory jump is dictated by the evaluation of a hopping probability, which is determined by the state amplitudes propagated along the trajectory. ${ }^{[4]}$

Despite its success, trajectory surface hopping cannot be formally derived from first-principles. It is not clear at first glance what are the consequences related to the use of independent (instead of correlated) trajectories and which role such approximation could play in the dynamics of systems when more than few degrees of freedom are considered. In addition, it is important to point out that, even though some quantum nuclear effects like the branching of the nuclear wavepackets are successfully described by TSH, others like (de)coherence and tunneling are inaccessible due to the use of classical trajectories.

One possible way to account for an accurate quantum propagation of the nuclei within a nonadiabatic molecular dynamics scheme is to employ quantum (or Bohmian) trajectories. [5] Those trajectories emerge from a transformation of the timedependent Schrödinger equation based on a polar representation of the complex nuclear wavefunction (see Section 2 below). Recognizing that the equation of motion for the phase of the nuclear wavepacket can be interpreted as an extended HamiltonJacobi equation, a Newton-like expression can be derived for the propagation of infinitesimally small volume elements of the configuration space (fluid elements), giving raise to a swarm of correlated quantum trajectories. In the synthetic formulation, ${ }^{[6]}$ the quantum trajectory method has been exploited to address challenging quantum dynamics problems in low dimensional model systems (see ref. [7] for an extended presentation of quantum trajectory methods). More generally, the Bohmian formulation of quantum mechanics offers an alternative solution to the interpretation of phenomena like wavepacket collisions and interferences. ${ }^{[8]}$ It is important to mention that in addition to the method developed in ref. [9] and presented in this work, other alternative trajectory-based solutions of the nonadiabatic quantum dynamics are presented in the literature that are however based on the diabatic representation of the electronic states. ${ }^{[10]}$

The method that we have developed (NABDY, NonAdiabatic Bohmian DYnamics) combines the use of quantum trajectories with nonadiabatic dynamics and therefore fully preserves the quantum nature of the nuclear dynamics. Its derivation is rigorous and the implementation only rests on controllable numerical approximations. In addition, this technique employs the adiabatic representation of the electronic states, which makes it suitable for an "on-the-fly" implementation, where potential energy surfaces, nuclear forces, and nonadiabatic couplings are evaluated at each time step using the desired electronic structure approach.

\section{Theory}

Starting from the time-dependent Schrödinger equation for a molecular system,

$$
\hat{H} \Psi(\boldsymbol{r}, \boldsymbol{R}, t)=i \hbar \frac{\partial}{\partial t} \Psi(\boldsymbol{r}, \boldsymbol{R}, t),
$$

we can use the Born-Huang Ansatz, ${ }^{[11]}$

$$
\Psi(\boldsymbol{r}, \boldsymbol{R}, t)=\sum_{I}^{\infty} \Phi_{I}(\boldsymbol{r} ; \boldsymbol{R}) \Omega_{I}(\boldsymbol{R}, t)
$$

to describe the total molecular wavefunction $\Psi(\boldsymbol{r}, \boldsymbol{R}, t)$. Here, $\boldsymbol{r}$ is a collective variable for all electronic positions and $\boldsymbol{R}$ those for the nuclei. $\Phi_{I}(\boldsymbol{r} ; \boldsymbol{R})$ is the $I^{\text {th }}$ solution of the time-independent electronic Schrödinger equation, and $\Omega_{\mathrm{I}}(\boldsymbol{R}, t)$ can be interpreted as the corresponding nuclear wavefunction. After inserting Eqn. (2) into Eqn. (1) and using the polar representation for a complex wavefunction

$$
\Omega_{J}(\boldsymbol{R}, t)=A_{J}(\boldsymbol{R}, t) \exp \left[\frac{i}{\hbar} S_{J}(\boldsymbol{R}, t)\right]
$$

we obtain the following equations of motion for the nuclear phase $S_{J}(\boldsymbol{R}, t)$ and amplitude $A_{J}(\boldsymbol{R}, t)$ (both real field),

$$
H_{J I}(\boldsymbol{R})=\int \Phi_{J}^{*}(\boldsymbol{r} ; \boldsymbol{R}) \hat{\mathcal{H}}_{e l} \Phi_{I}(\boldsymbol{r} ; \boldsymbol{R}) d \boldsymbol{r}
$$

The quantities $\mathbf{d}_{\mathrm{JI}}^{\gamma}$ and $\mathrm{D}_{\mathrm{JI}}^{\gamma}$ are the first and second order nonadiabatic couplings, respectively,

$$
\begin{aligned}
& \boldsymbol{d}_{J I}^{\gamma}(\boldsymbol{R})=\int \Phi_{J}^{*}(\boldsymbol{r} ; \boldsymbol{R}) \nabla_{\gamma} \Phi_{I}(\boldsymbol{r} ; \boldsymbol{R}) d \boldsymbol{r} \\
& D_{J I}^{\gamma}(\boldsymbol{R})=\int \Phi_{J}^{*}(\boldsymbol{r} ; \boldsymbol{R}) \nabla_{\gamma}^{2} \Phi_{I}(\boldsymbol{r} ; \boldsymbol{R}) d \boldsymbol{r}
\end{aligned}
$$

Within the Hamilton-Jacobi formulation of mechanics, the relation between momentum and phase of the nuclear wavepacket,

$$
\nabla_{\beta} S_{J}(\boldsymbol{R}, t)=\boldsymbol{P}_{\beta}^{J},
$$

gives rise to a Newton-like equation for the nuclear motion ${ }^{[9]}$

$$
\begin{aligned}
& M_{\beta} \frac{d^{2} \boldsymbol{R}_{\beta}}{\left(d t^{J}\right)^{2}}= \\
& -\nabla_{\beta}\left[E_{e l}^{J}(\boldsymbol{R})+\mathcal{Q}_{J}(\boldsymbol{R}, t)+\mathcal{D}_{J}(\boldsymbol{R}, t)\right]
\end{aligned}
$$

where the definition of the time derivative in a Lagrangian frame

$$
\begin{aligned}
& d / d t^{J}= \\
& \partial / \partial t+\sum_{\gamma} \nabla_{\gamma} S_{J}(\boldsymbol{R}, t) / M_{\gamma} \cdot \nabla_{\gamma}
\end{aligned}
$$

is used.

In addition to the 'classical' potential $E_{e l}{ }^{J}(\boldsymbol{R})$, the nuclei will feel a quantum potential $Q_{J}(\boldsymbol{R}, t)$, which describes adiabatic quantum nuclear effects, and a nonadiabatic quantum potential $D_{J}(\boldsymbol{R}, t)$ responsible for the couplings between electronic states.

At this stage, it is important to point out that no approximation has been made to derive the set of working equations (Eqns. (8), (9) and (12)).

$$
\begin{aligned}
-\frac{\partial S_{J}(\boldsymbol{R}, t)}{\partial t} & =\sum_{\gamma} \frac{1}{2 M_{\gamma}}\left(\nabla_{\gamma} S_{J}(\boldsymbol{R}, t)\right)^{2}+E_{J}^{e l}(\boldsymbol{R})-\sum_{\gamma} \frac{\hbar^{2}}{2 M_{\gamma}} \frac{\nabla_{\gamma}^{2} A_{J}(\boldsymbol{R}, t)}{A_{J}(\boldsymbol{R}, t)} \\
& +\sum_{\gamma I} \frac{\hbar^{2}}{2 M_{\gamma}} D_{J I}^{\gamma}(\boldsymbol{R}) \frac{A_{I}(\boldsymbol{R}, t)}{A_{J}(\boldsymbol{R}, t)} \Re\left[e^{i \phi(\boldsymbol{R}, t)}\right]-\sum_{\gamma, I \neq J} \frac{\hbar^{2}}{M_{\gamma}} \boldsymbol{d}_{J I}^{\gamma}(\boldsymbol{R}) \frac{\nabla_{\gamma} A_{I}(\boldsymbol{R}, t)}{A_{J}(\boldsymbol{R}, t)} \Re\left[e^{i \phi(\boldsymbol{R}, t)}\right] \\
& +\sum_{\gamma, I \neq J} \frac{\hbar}{M_{\gamma}} \boldsymbol{d}_{J I}^{\gamma}(\boldsymbol{R}) \frac{A_{I}(\boldsymbol{R}, t)}{A_{J}(\boldsymbol{R}, t)} \nabla_{\gamma} S_{I}(\boldsymbol{R}, t) \Im\left[e^{i \phi(\boldsymbol{R}, t)}\right]
\end{aligned}
$$

and

$$
\begin{aligned}
\frac{\partial A_{J}(\boldsymbol{R}, t)}{\partial t}= & -\sum_{\gamma} \frac{1}{M_{\gamma}} \nabla_{\gamma} A_{J}(\boldsymbol{R}, t) \nabla_{\gamma} S_{J}(\boldsymbol{R}, t)-\sum_{\gamma} \frac{1}{2 M_{\gamma}} A_{J}(\boldsymbol{R}, t) \nabla_{\gamma}^{2} S_{J}(\boldsymbol{R}, t) \\
& +\sum_{\gamma I} \frac{\hbar}{2 M_{\gamma}} D_{J I}^{\gamma}(\boldsymbol{R}) A_{I}(\boldsymbol{R}, t) \Im\left[e^{i \phi(\boldsymbol{R}, t)}\right]-\sum_{\gamma, I \neq J} \frac{\hbar}{M_{\gamma}} \boldsymbol{d}_{J I}^{\gamma}(\boldsymbol{R}) \nabla_{\gamma} A_{I}(\boldsymbol{R}, t) \Im\left[e^{i \phi(\boldsymbol{R}, t)}\right] \\
& -\sum_{\gamma, I \neq J} \frac{1}{M_{\gamma}} \boldsymbol{d}_{J I}^{\gamma}(\boldsymbol{R}) A_{I}(\boldsymbol{R}, t) \nabla_{\gamma} S_{I}(\boldsymbol{R}, t) \Re\left[e^{i \phi(\boldsymbol{R}, t)}\right],
\end{aligned}
$$

where

$$
\phi(\boldsymbol{R}, t)=\frac{1}{\hbar}\left[S_{I}(\boldsymbol{R}, t)-S_{J}(\boldsymbol{R}, t)\right],
$$




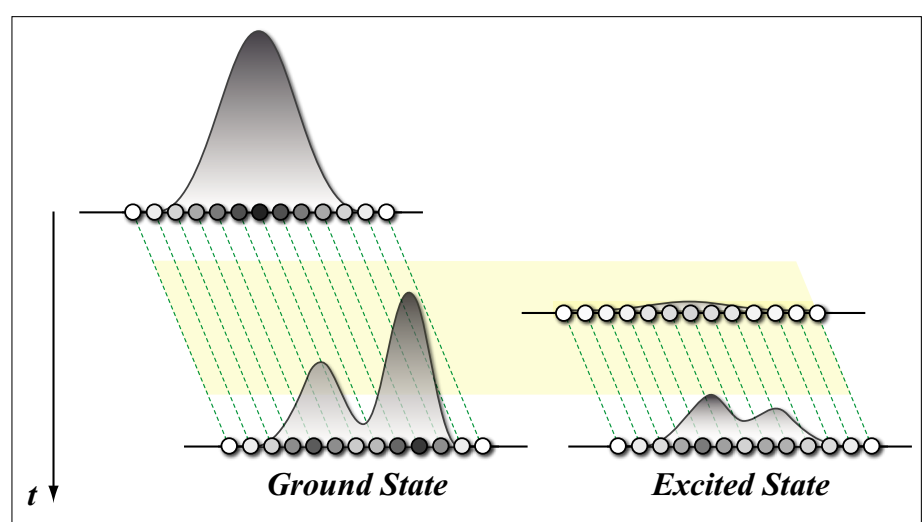

Fig. 2. Schematic representation of the NABDY scheme. Left (right): evolution of the nuclear wavepacket in state 1 (state 2). The vertical axis represents time and the light background indicates a region of strong nonadiabatic coupling between the two states.

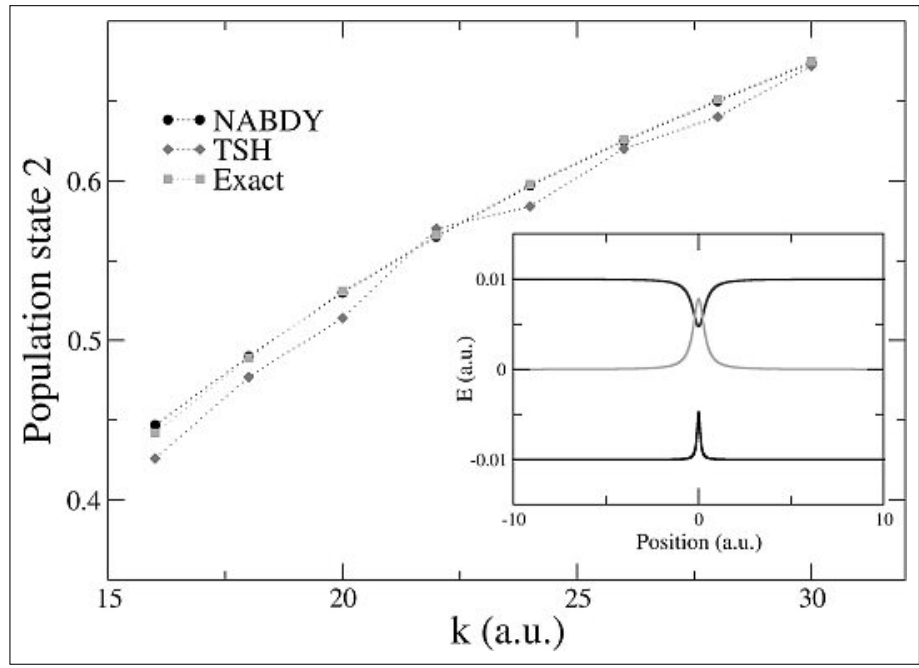

Fig. 3. 1D nonadiabatic system based on Tully model 1. Comparison between NABDY, the exact propagation and TSH is presented for different initial momenta of the nuclear wavepacket. Inset: lower and upper black lines represent the ground and first excited state energies, whereas the strength of the nonadiabatic coupling is plotted in gray.

Numerically, we start from an initial nuclear wavepacket at time $\mathrm{t}=0$, which is represented by an ensemble of fluid elements (small volume elements of the configuration space, see Fig. 2). In fact, the fluid elements can be seen as correlated 'grid points', each carrying an amplitude, a phase, and a velocity. The integration of the NABDY equation of motions consists in the simultaneous solution of the differential equations for the phases (Eqn. (4)), the amplitudes (Eqn. (5)), and the Newtonlike equations for the fluid elements (Eqn. (11)). During the adiabatic propagation of the quantum trajectories, nonadiabatic couplings are monitored and, when their strength exceeds a given threshold, the dynamics on the coupled states is started (Fig. 2). In this way, not only trajectories on one state are correlated, but also among different electronic states. For numerical details, see ref. [9].

\section{Numerical Applications}

In this section, we briefly summarize some of our first results obtained with the current numerical implementation of NABDY. ${ }^{[9]}$

In the first example, a simple one-dimensional system based on Tully model ${ }^{\left[{ }^{[4]}\right.}$ was employed to assess the accuracy of the numerical scheme. As shown in Fig. 3, the population of the second state obtained using NABDY is in full agreement (always within $0.1 \%$ ) with an exact propagation applying the time-dependent Schrödinger equation. ${ }^{[12]}$ Interestingly, despite its approximations, also TSH performs relatively well in this last test case. In particular, the independent trajectory approximation that lies at the core of TSH seams not to play an important role, probably due to the weak coupling between the wavepacket on the two potential energy curves.

Finally, we applied NABDY to the study of a simple molecular process, name- ly the collision of an $\mathrm{H}$ atom with a $\mathrm{H}_{2}$ molecule. In this case, PESs and nonadiabatic couplings are computed on-the-fly using DFT and LR-TDDFT in LDA approximation for the exchange-correlation functional, as implemented in the plane-wave code CPMD. [13] The calculation of the NACVs within the LR-TDDFT ${ }^{[14]}$ framework was the subject of a recent investigation, where we showed how the use of auxiliary manyelectron wavefunctions based on occupied and virtual Kohn-Sham orbitals can simplify the evaluation of NACVs between ground state and excited state ${ }^{[15]}$ and between a pair of excited states. ${ }^{[16]}$ Here we present a particular case (see Fig. 4), in which the $\mathrm{H}$ atom is directed almost perpendicular to the $\mathrm{H}_{2}$ bond axis with two different initial momenta, $\mathrm{k}=75$ and $\mathrm{k}=$ 150 a.u. The $\mathrm{H}_{2}$ bond is considered fixed, simplifying the nuclear propagation. In this case, we observed a population transfer to the first excited state of respectively $27.9 \%$ and $31.4 \%$ for each momentum.

\section{Numerical Challenges}

There are several numerical issues that hamper the efficient implementation of NABDY in $a b$ initio MD codes. As it can be seen in Eqn. (4), many of the terms in the right hand side are inversely proportional to the nuclear amplitude on a given state. This clearly induces numerical instabilities when nodes are formed on the wavepacket. Different schemes have been proposed to cure this problem ${ }^{[7]}$ in the adiabatic propagation. The addition of nonadiabatic terms makes the instabilities even larger as can be observed in Fig. 3 in the case of small momentum ( $\mathrm{k}=16$ a.u.).

In addition, it is also important to point out that the spatial derivatives in Eqns. (4), (5), and (11) need formally to be evaluated in a $3 \mathrm{M}$ dimensional space, where $\mathrm{M}$ is the number of nuclei of the molecular system of interest. Even though different ways have been proposed to compute these

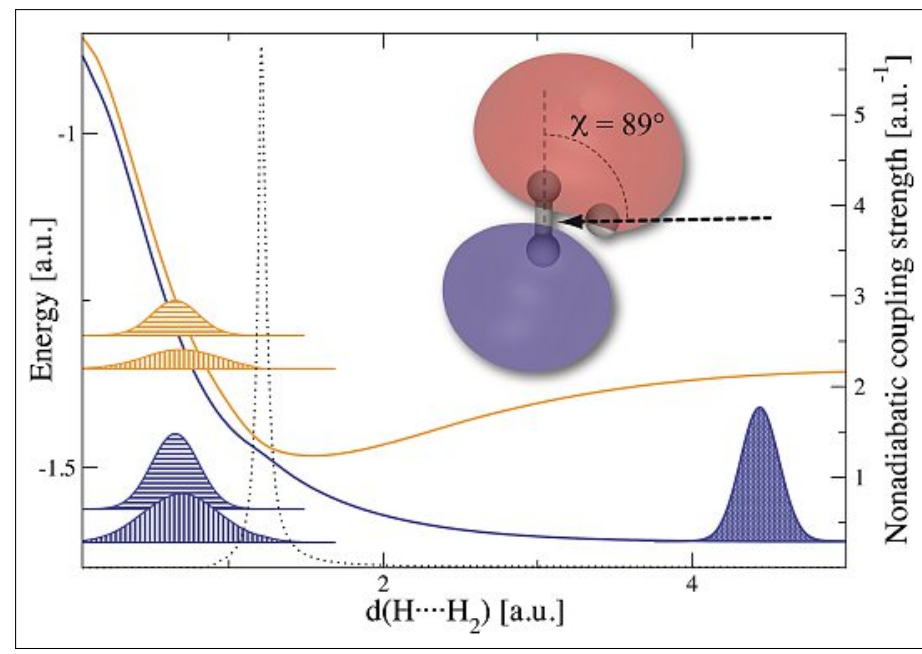

Fig. 4. Collision of a $\mathrm{H}$ atom with $\mathrm{a}_{2}$ molecule. PESs (blue: ground state, orange: first excited state) and nonadiabatic couplings (black dotted line) are computed via DFT/LR-TDDFT. The inset shows the LUMO orbital of the system close to the avoided crossing. 
derivatives on a regular grid, they can still constitute a potential bottleneck for the implementation of this type of dynamics in the full, unconstrained, configuration space.

\section{Conclusions}

We have presented a new algorithm to perform nonadiabatic quantum dynamics using Bohmian trajectories. The method, called NABDY, can be performed on-thefly when combined with an efficient electronic structure method for the evaluation of the energies, forces, and nonadiabatic couplings. Even though in low dimensions nodes of the wavepacket can bring important numerical instabilities, we have presented systems for which NABDY can reproduce with great accuracy results obtained using exact quantum dynamics.

\section{Acknowledgements}

The authors thank Giovanni Ciccotti, Jiří Vaníček, Sara Bonella, Tomáš Zimmermann, Jérôme F. Gonthier, Thomas J. Penfold, and Felipe Franco de Carvalho for their useful comments. COST action CM0702, Swiss NSF grant 200020-130082, and NCCR-MUST are acknowledged for fundings.

Received: January 24, 2012

[1] D. Marx, J. Hutter, 'Ab initio molecular dynamics', Cambridge University Press, Cambridge, 2009.

[2] A. W. Jasper, S. Nangia, C. Zhu, D. G. Truhlar, Acc. Chem. Res. 2006, 39, 101; T. Yonehara, S. Takahashi, K. Takatsuka, J. Chem. Phys. 2009, 130, 214113; K. Takatsuka, Int. J. Quant. Chem. 2009, 109, 2131.

[3] M. H. Beck, A. Jäckle, G. A. Worth, H.-D. Meyer, Phys. Rep. 2000, 324, 1; R. S. Minns, D. S. N. Parker, T. J. Penfold, G. A. Worth, H. H. Fielding, Phys. Chem. Chem. Phys. 2010, 12, 15607.

[4] J. C. Tully, J. Chem. Phys. 1990, 93, 1061.

[5] D. Bohm, Phys. Rev. 1952, 85, 166; D. Bohm, Phys. Rev. 1952, 85, 180; T. Takabayasi, Prog. Theor. Phys. 1952, 8, 143.

[6] C. L. Lopreore, R. E. Wyatt, Phys. Rev. Lett. 1999, 82, 5190 .

[7] R. E. Wyatt, 'Quantum dynamics with trajectories: Introduction to quantum hydrodynamics', Springer, Interdisciplinary applied mathematics, 2005.

[8] A. S. Sanz, S. Miret-Artés, J. Phys. A: Math. Theor. 2008, 41, 435303.

[9] B. F. E. Curchod, I. Tavernelli, U. Rothlisberger, Phys. Chem. Chem. Phys. 2011, 13, 3231.
[10] R. E. Wyatt, C. L. Lopreore, G. Parlant, J. Chem. Phys. 2001, 114, 5113; C. L. Lopreore, R. E. Wyatt, J. Chem. Phys. 2002, 116, 1228; S. Garashchuk, V. A. Rassolov, G. C. Schatz, J. Chem. Phys. 2005, 123, 174108; B. Poirier, G. Parlant, J. Phys. Chem. A 2007, 111, 10400

[11] M. Born, K. Huang, 'Dynamical Theory of Crystal Lattices', Clarendon, Oxford, 1954.

[12] The exact wavepacket propagation was performed in the adiabatic representation using the Fourier propagation method (courtesy of J. Vaníček and T. Zimmermann).

[13] CPMD. Copyright IBM Corp 1990-2001, Copyright MPI für Festkörperforschung Stuttgart, 1997-2001. http://www.cpmd.org.

[14] E. Runge, E. K. U. Gross, Phys. Rev. Lett. 1984 52, 997; M. E. Casida, in 'Recent Advances in Density Functional Methods', Ed. D. P. Chong, World Scientific, Singapore, 1995, p.155; M. Petersilka, U. J. Gossmann,, E. K. U. Gross, Phys. Rev. Lett. 1996, 76, 1212; H. Appel, E. K. U. Gross, K. Burke, Phys. Rev. Lett. 2003, 90, 043005.

[15] E. Tapavicza, I. Tavernelli, U. Rothlisberger, Phys. Rev. Lett. 2007, 98, 023001.; I. Tavernelli, E. Tapavicza, U. Rothlisberger, J. Chem. Phys. 2009, 130, 124107; I. Tavernelli, B. F. E. Curchod, U. Rothlisberger, J. Chem. Phys. 2009, 131, 196101 .

[16] I. Tavernelli, B. F. E. Curchod, A. Laktionov, U. Rothlisberger, J. Chem. Phys. 2010, 133 194104. 